\title{
Obesity: focus on ongoing multidisciplinary and comprehensive research
}

\author{
Paolo Sbraccia ${ }^{1} \cdot$ Enzo Nisoli ${ }^{2} \cdot$ Rocco Barazzoni $^{3}$
}

Received: 6 November 2017 / Accepted: 7 November 2017 / Published online: 11 December 2017

๑) Springer International Publishing AG, part of Springer Nature 2017

We are pleased to announce the launch of a new Web-edited "Topical Collection" that will gather review articles addressing the most relevant aspects of obesity, and which will be contributed by experts in the field. They will be published in Eating and Weight Disorders-Studies on Anorexia, Bulimia and Obesity on behalf of the Italian Society of Obesity (SIO) and, therefore, named "Italian Society of Obesity (SIO) Reviews". This series aims to collect invited articles from the leading Italian experts on obesity and related disorders, thus strengthening the already fruitful collaboration between the Journal and our Society. In fact, over the past few years, in Eating and Weight Disorders-Studies on Anorexia, Bulimia and Obesity has become the official Journal of SIO. Eating and Weight Disorders-Studies on Anorexia, Bulimia and Obesity has reached an authoritative position in the field of eating disorders, particularly in anorexia and bulimia nervosa, and in related disorders. Now, it will support more intensively also the obesity and nutrition research, topics after all already well explored by several papers published in recent years.

Open and without time limits, this collection will guest articles from invited international scientists, aimed to provide a geographically wide perspective and cover the broadest range of expertise. Our purpose is that this forum becomes an opportunity for interaction of different specialties, research and clinical networking, highlighting of priorities, and searching for shared evidence-based hypothesis and hopefully solutions of health policy and interventions. Articles will be selected and organized in diverse thematic subjects, focusing to the most significant topics in obesity, reviewing the most exciting progress in molecular and clinical research, and serving as an immediate and upto-date resource for the readers.

"SIO Reviews" will be guest-edited by Paolo Sbraccia and Enzo Nisoli, already Associate Editors in Eating and Weight Disorders-Studies on Anorexia, Bulimia and Obesity, and Rocco Barazzoni, who currently serves as coordinator of the SIO Scientific Committee.

In conclusion, the papers in this Topical Collection are intended as a small cross-section of the multidisciplinary and comprehensive research ongoing in the area of obesity.

We hope that this collection will offer the readers a valuable tool to overview the most recent advances quickly, seek research collaborations, and increase their expertise in the obesity field.

\section{Compliance with ethical standards}

Conflict of interest The authors declare they have no conflict of interest.

This article is part of the topical collection on Italian Society of Obesity's Reviews.

Paolo Sbraccia

sbraccia@med.uniroma2.it

1 Department of Systems Medicine, Medical School, University of Rome Tor Vergata, Via Montpellier 1, 00133 Rome, Italy

2 Department of Medical Biotechnology and Translational Medicine, Center for Study and Research on Obesity, University of Milan, Milan, Italy

3 Department of Medical, Surgical and Health Sciences, University of Trieste, Trieste, Italy 